\title{
Diffuse Ionised Gas in Edge-on Galaxies
}

\author{
Richard J. Rand \\ Department of Physics and Astronomy, University of \\ New Mexico, Albuquerque, NM 87131, USA \\ rjr@gromit.phys.unm.edu \\ Received 1997 August 1, accepted 1997 December 15
}

\begin{abstract}
We review observations of diffuse ionised gas (DIG) in edge-on spiral galaxies. Deep imaging has revealed a variety of morphologies for 'extraplanar' (above the HII region layer) or 'halo' DIG: some show widespread prominent layers of truly diffuse gas and filamentary structures, others show just one patch or a few patches of extraplanar emission, while still others show no detectable extraplanar DIG at all. The key galactic property which appears to govern the prominence of the DIG layer is the star-formation activity. Smaller-scale connections between DIG and local star formation activity can be seen in some of the galaxies.

DIG halos are also related to radio continuum, X-ray and $\mathrm{HI}$ halos. Those galaxies with the most prominent DIG layers also have the most prominent radio halos. In NGC 891, there are spatial correlations between extraplanar DIG, radio emission, X-ray emission and HI, and these in turn are related to the underlying disk star-formation activity.

Progress on identifying the sources of ionisation and heating of the gas has come from spectroscopy, imaging in different emission lines, and Fabry-Perot observations. By comparing with theoretical predictions, much evidence is found for photoionisation by disk stars as the primary ionisation and heating mechanism, but recent observations are beginning to point towards the need for additional sources of ionisation and/or heating.
\end{abstract}

Keywords: galaxies: ISM — galaxies: halos — galaxies: spiral — galaxies: structure radiative transfer

\section{Introduction}

With the advent of the AAO/UKST Galactic Plane $\mathrm{H} \alpha$ survey and the several other Galactic surveys discussed in this issue, an opportunity arises for progress in understanding the origin, structure, and ionisation of DIG through the comparison of these surveys with observations of external edge-on galaxies. These galaxies offer the advantage of allowing the entire DIG layer to be quickly imaged (albeit at relatively low linear resolution), while avoiding the difficulty inherent in Milky Way observations of translating angular heights of emission to physical heights. Studies of the diffuse ionised layer of the Milky Way will allow, for example, the vertical structure of the layer and the dependence of line ratios on height from the Galactic plane and on the underlying disk environment to be compared with results from external edge-ons. The importance of DIG as an ISM component and the leverage it provides on understanding the ISM as a whole is discussed elsewhere in this issue by Reynolds, Ferrara, Elmegreen and others. The goal of this review is to summarise our understanding of DIG in edge-on galaxies at the time when these surveys are getting under way.

\section{DIG Morphology}

NGC 891, having an optical appearance similar to that of the Milky Way, and being nearby $(D=9 \cdot 5$ Mpc) and almost perfectly edge on (Swaters 1994), was the first target in the search for extraplanar DIG layers (Rand, Kulkarni \& Hester 1990; Dettmar 1990). There, a bright, widespread DIG halo was found, showing truly diffuse emission as well as several quasi-vertical filaments of height $2-3 \mathrm{kpc}$. The halo could be traced up to $z=3.5 \mathrm{kpc}$, but recent deep spectroscopy (Rand 1997; see below) shows that it extends to at least $z=5.5 \mathrm{kpc}$. Furthermore, the spectra confirm the earlier result that there are two vertical components to the emission, with electron density scale heights (assuming that the gas temperature and filling factor are constant with $z$ ) of $1 \mathrm{kpc}$ and 5-6 kpc. The emission is concentrated to within about $R=8 \mathrm{kpc}$ of the centre of the galaxy, with the brightest region at about $R=5-8 \mathrm{kpc}$ on the north side and a rather sharp cutoff beyond this region. Disk star formation, as traced by [C II] $158 \mu \mathrm{m}$ emission (Madden et al. 1992), shows a radial profile with very similar asymmetries (Rand 1994).

Almost without exception, the $\mathrm{H} \alpha$ filaments have their footprint in bright HII regions in the disk 
Table 1. Comparison of extraplanar DIG properties with traces of star formation

\begin{tabular}{|c|c|c|c|c|c|c|c|}
\hline Galaxy & Type & Dist. (Mpc) & $\begin{array}{l}\text { Adopted } \\
\text { incl. }^{\mathrm{A}}\end{array}$ & $\begin{array}{c}\text { DIG } \\
\text { morphol. }\end{array}$ & $\begin{array}{c}L_{F I R}{ }^{\mathrm{B}} \\
\left(10^{43} \mathrm{erg} \mathrm{s}^{-1}\right)\end{array}$ & $\begin{array}{c}L_{F I R} / D_{25}^{2} \\
\left(10^{40} \mathrm{erg} \mathrm{s}^{-1} \mathrm{kpc}^{-2}\right)\end{array}$ & DIG ref. \\
\hline NGC 3079 & $\mathrm{Sc}$ & $17 \cdot 3$ & 84 & Bright diffuse + filaments & 14 & $8 \cdot 9$ & (1) \\
\hline NGC 5775 & $\mathrm{Sc}$ & $26 \cdot 7$ & 85 & Many bright fil. & $8 \cdot 6$ & $8 \cdot 1$ & $(2)$ \\
\hline Milky Way & $\mathrm{Sb}$ & $\ldots$ & $\ldots$ & Diff. + fil. & $3 \cdot 8^{\mathrm{C}}$ & $3 \cdot 0$ & $(3)$ \\
\hline NGC 891 & $\mathrm{Sb}$ & $9 \cdot 5$ & 89 & Bright diff.+fil. & $3 \cdot 1$ & $2 \cdot 2$ & (4) \\
\hline NGC 4013 & $\mathrm{Sbc}$ & $17 \cdot 0$ & 90 & Faint diff.+fil. & $1 \cdot 4$ & $2 \cdot 6$ & $(5)$ \\
\hline NGC 4631 & $\mathrm{Sd}$ & $7 \cdot 5$ & 85 & Bright diff., patchy & $2 \cdot 0$ & $1 \cdot 8$ & (6) \\
\hline NGC 55 & $\mathrm{Sm}$ & $7 \cdot 5$ & 79 & Bright diff.+fil. & $0 \cdot 15$ & $0 \cdot 5$ & $(7)$ \\
\hline NGC 4302 & $\mathrm{Sc}$ & $16 \cdot 8$ & 90 & Faint diff. & $<1 \cdot 2^{\mathrm{D}}$ & $<2 \cdot 3$ & (5) \\
\hline NGC 5907 & $\mathrm{Sc}$ & $14 \cdot 9$ & 86 & No halo DIG & $2 \cdot 0$ & $0 \cdot 8$ & $(5)$ \\
\hline UGC 10288 & $\mathrm{Sc}$ & $31 \cdot 5$ & 86 & 3 or 4 plumes & $0 \cdot 8$ & $0 \cdot 4$ & $(5)$ \\
\hline NGC 4565 & $\mathrm{Sb}$ & $9 \cdot 7$ & 86 & No halo DIG & $0 \cdot 5$ & $0 \cdot 3$ & (6) \\
\hline NGC 5746 & $\mathrm{Sb}$ & $29 \cdot 4$ & 86 & No halo DIG & $1 \cdot 4$ & $0 \cdot 2$ & (5) \\
\hline NGC 4244 & Scd & $3 \cdot 1$ & 88 & No halo DIG & $0 \cdot 1$ & $0 \cdot 01$ & $(8)$ \\
\hline NGC 5023 & Scd & 6 & 87 & 1 or 2 plumes, 1 faint patch & $<0 \cdot 01^{\mathrm{E}}$ & $<0 \cdot 09$ & (5) \\
\hline NGC 4217 & $\mathrm{Sb}$ & $17 \cdot 0$ & 86 & 2 faint patches & $<0 \cdot 08^{\mathrm{E}}$ & $<0 \cdot 12$ & (5) \\
\hline UGC 4278 & $\mathrm{Sd}$ & $10 \cdot 6$ & $87-90$ & 2 plumes+patches & $<0 \cdot 03^{\mathrm{E}}$ & $<0 \cdot 04$ & (5) \\
\hline NGC 4762 & So & $16 \cdot 8$ & 90 & No halo DIG & $<0 \cdot 08^{\mathrm{E}}$ & $<0 \cdot 15$ & (5) \\
\hline
\end{tabular}

Values of $D_{25}$ are from de Vaucouleurs et al. (1991).

References: (1) Veilleux et al. (1995); (2) Dettmar (1992); (3) Reynolds (1993); (4) Rand, Kulkarni \& Hester (1990), Dettmar (1990); (5) Rand (1996); (6) Rand, Kulkarni \& Hester (1992); (7) Ferguson, Wyse \& Gallager (1996); (8) Walterbos (1991). A References for inclinations of previously observed galaxies: NGC 3079 (Irwin \& Seaquist 1991), NGC 5775 (Dettmar 1992), NGC 891 (Swaters 1994), NGC 4631 and NGC 4565 (Rand, Kulkarni \& Hester 1992), NGC 4244 (Walterbos 1991).

B For external galaxies, from Rice et al. (1988) where possible; otherwise from the IRAS Cataloged Galaxies and Quasars.

C From Cox \& Mezger (1989).

$\mathrm{D}$ The IRAS source is compact and between NGC 4302 and its companion NGC 4298 . These are separated by $2.4^{\prime}$, so the IRAS source is probably a blend of emission from both galaxies.

$\mathrm{E}$ Not detected by IRAS. These are estimated $3 \sigma$ upper limits assuming that the detection limit in these fields was typical of the IRAS observations. None of these galaxy fields was missed by IRAS.

(Rand 1994). Some are quite narrow and may be walls of chimneys (Norman \& Ikeuchi 1989), others are broader and may be 'ionisation cones': regions where relatively exposed $\mathrm{O}$ and $\mathrm{B}$ stars have been able to ionise large volumes of diffuse gas above and below the midplane (Miller \& Cox 1993; Dove \& Shull 1994). In the case of the former, the connection with HII regions suggests that star formation persists in these $\sim 1 \mathrm{kpc}$ regions of disk for at least as long as the $\sim 10^{7}$ yr dynamical time (Norman \& Ikeuchi 1989) to produce the chimneys. This connection between filaments and HII regions is also seen in NGC 2188 (Domgörgen, Dahlem \& Dettmar 1996) and NGC 55 (Ferguson, Wyse \& Gallagher 1996; Wyse 1998, this issue p. 118).

Since these observations, over a dozen edge-ons have been observed with similar sensitivity, with the result that bright, extended DIG layers as in NGC 891 are the exception rather than the rule (Table 1). Other galaxies with bright layers include NGC 4631 (although only above the central few kpc; Rand, Kulkarni \& Hester 1992), NGC 5775 (Dettmar 1992), and NGC 3079 (Veilleux, Cecil \& Bland-Hawthorn 1995). However, most galaxies seem to show only one or a few isolated patches or filaments of emission above the HII region layer, while some show no extraplanar DIG at all (Rand 1996). The prominence of the extraplanar DIG emission is related to the star formation activity in the disk. Insofar as far-infrared luminosity $\left(L_{F I R}\right)$ is a tracer of star-formation activity (debate continues on what fraction of the emission comes from dust heated by the ISRF; however, one cannot use $\mathrm{H} \alpha$ emission as a star-formation tracer because of the severe extinction problem), then the galaxies with high surface densities of star formation $\left(L_{F I R} / D_{25}^{2}\right)$ generally have the brightest and most prominent extraplanar DIG layers (Rand 1996). This general connection is strengthened by the aforementioned correlation of halo DIG and disk [C II] emission profiles in NGC 891 and the filament-HII region associations, and it is probably more accurate to say that active star-forming regions tend to have associated extraplanar DIG.

Current directions in this area include attempts at better characterisation of the background emission to allow heavier smoothing, thus making possible searches for emission at much fainter levels. Examples are the 'shift and stare' technique (Donahue, Aldering \& Stocke 1995) and 'charge shuffling' (BlandHawthorn \& Jones 1998, this issue p. 44). In this way, one can hope to determine the extent of halos at low column densities and their possible relevance as QSO absorption-line systems.

\section{DIG and Other Gaseous Halos}

The galaxies with the brightest extraplanar DIG layers also tend to have bright and extended radio continuum halos (Rand 1996). Examples are NGC 891 (e.g. Dahlem, Dettmar \& Hummel 1994), NGC 
4631 (e.g. Golla \& Hummel 1994), and NGC 5775 (Dettmar 1992; Duric, Irwin \& Bloemen 1996). At the other end of the scale, NGC 4244, with no indications of extraplanar DIG (Walterbos \& Braun 1996), was not detected at all in radio continuum (Hummel, Sancisi \& Ekers 1984). Dettmar (1992) reports an association between filaments of radio and $\mathrm{H} \alpha$ emission in the halo of NGC 5775. One might expect an $\mathrm{H} \alpha$-radio correlation if magnetic fields associated with the thermal gas serve to confine the cosmic-ray electrons as they try to escape the disk (N. Duric, personal communication). Observational connections between radio halo and star-formation distributions in edge-ons have also been discussed by Dahlem, Lisenfeld \& Golla (1995). Most of the galaxies in Table 1 have not been observed with sufficient sensitivity and resolution to search for a radio halo.

Diffuse X-ray halo emission is also difficult to detect and has been searched for in only a few of these edge-ons with ROSAT. NGC 891 (Bregman \& Pildis 1994; Bregman \& Houck 1997) and NGC 4631 (Wang et al. 1994) are notable for having relatively bright X-ray halos occupying about the same radial range as the $\mathrm{H} \alpha$ halos. However, this trend is not perfect: patches of diffuse halo X-ray emission have been found in NGC 4565 (Vogler, Pietsch \& Kahabka 1996), which shows no extraplanar DIG.

NGC 891 has a very thick disk of $21 \mathrm{~cm}$ emission, and a careful study of the origin of this high- $z$ gas has been carried out by Swaters (1994). The kinematic information allows inner-disk high- $z$ gas, which may be accelerated by supernova activity, to be distinguished from outer-disk gas, which may be due to warps and flares. The result is that the most prominent inner-disk high- $z$ emission is coincident with the brightest region of halo DIG and radio emission, sitting above the brightest [C II] $158 \mu \mathrm{m}$ emission in the disk (all at about $R=5-8 \mathrm{kpc}$ on the north side). This HI is detected up to $2.5 \mathrm{kpc}$ above the disk. There does not seem to be a good correlation between individual $\mathrm{HI}$ and $\mathrm{H} \alpha$ filaments, however, although the lower $\left(20^{\prime \prime}\right)$ resolution of the HI may hamper the comparison. Other bright star-forming edge-ons (NGC 3079, NGC 5775) show HI arcs, shells and filaments extending several $\mathrm{kpc}$ from the plane (Irwin \& Seaquist 1990; Irwin 1994). Connections between these and $\mathrm{H} \alpha$ features are not yet clear.

The emergent scenario, then, which needs to be tested further, is that star-formation activity, when sufficiently intense, leads to the elevation of gas from the thin star-forming layer, through chimneys and superbubbles, leading to halo DIG, $\mathrm{HI}$ and X-ray emission. Meanwhile, diffusion and possibly localised regions of convection of cosmic rays produced in supernovae (e.g. Duric, Irwin \& Bloemen 1996) produce cosmic ray halos, traceable through radio continuum emission.

\section{Emission Line Ratios as Diagnostics of Ionisation and Heating}

In the Reynolds layer, photoionisation by massive stars in the disk is considered the most likely primary ionisation mechanism (Reynolds 1998, this issue p. 14). The available energy easily satisfies that required to maintain the layer, and photoionisation models [e.g. Mathis 1986; Sokolowski 1994 (partially reproduced in Bland-Hawthorn, Freeman \& Quinn 1997)] have been the most successful in explaining the behaviour of line ratios (enhanced [S II] $\lambda \lambda 6716,6731 / \mathrm{H} \alpha$ and $[\mathrm{N} \mathrm{II}] \lambda 6583 / \mathrm{H} \alpha$, and very weak $[\mathrm{O} \mathrm{I}] \lambda 6300 / \mathrm{H} \alpha$, and $[\mathrm{O}$ III $] \lambda 5007 / \mathrm{H} \alpha$ relative to $\mathrm{HII}$ regions are due to dilution of the stellar radiation as it propagates away from the Galactic plane). However, there are observations that these models cannot explain, one of which is the weakness of $\mathrm{He} \mathrm{I} \lambda 5876 / \mathrm{H} \alpha$ (Reynolds \& Tufte 1995; Reynolds 1998), a ratio with a fairly straightforward interpretation in terms of the hardness of the ionising radiation [see also Heiles et al. (1996) for an even more extreme result from radio recombination lines]. The HeI emission is much weaker than expected from models, given the high $[\mathrm{N} \mathrm{II}] / \mathrm{H} \alpha$ and $[\mathrm{SII}] / \mathrm{H} \alpha$ values (see Domgörgen \& Mathis 1994). The forbidden lines are highly temperature-sensitive and their interpretation is complicated by issues of abundances, gas heating, and depletion of important coolants such as $\mathrm{Fe}, \mathrm{Si}$, and Ca (Sokolowski 1994). But regardless of the forbidden lines, the low $\mathrm{He} / / \mathrm{H} \alpha$ is problematic in itself and may indicate that we do not understand stellar atmospheres in the extreme UV well enough (see e.g. Cassinelli et al. 1995), and/or which spectral types of massive stars are most important for the ionisation (Reynolds \& Tufte 1995; Heiles et al. 1996).

It is also not clear to what degree other sources of ionisation contribute: shocks (which may play a role in irregulars; Martin 1996), turbulent mixing layers (Slavin, Shull \& Begelman 1993), cooling fountain gas (Shapiro \& Benjamin 1993) and decaying neutrinos (e.g. Sciama 1995).

In NGC 891, the run of $[\mathrm{NII}] / \mathrm{H} \alpha$ versus $z$ at $R=5 \mathrm{kpc}$ on the north side has been measured by Rand (1997). This shows a smooth increase with $z$, from 0.4 in the midplane to 1.4 at $z=2 \mathrm{kpc}$ (east side of midplane) and $z=4 \mathrm{kpc}$ (west side). Previous, less sensitive spectroscopy by Dettmar \& Schulz (1992) and Keppel et al. (1991) also showed an increase with $z$ for $[\mathrm{NII}] / \mathrm{H} \alpha$ as well as $[\mathrm{SII}] / \mathrm{H} \alpha$. The same trend in $[\mathrm{SII}] / \mathrm{H} \alpha$ was also seen in narrowband images (Rand, Kulkarni \& Hester 1990). The spatial behaviour is as expected in photoionisation models (the ratios rise with distance from the disk ionising sources as the radiation field is diluted) but values as high as 1.4 are difficult to explain (Sokolowski 1994). Note that in M31 
(Walterbos 1998, this issue p. 99) and the Milky Way, $[\mathrm{N} \mathrm{II}] / \mathrm{H} \alpha$ is roughly constant at only $0 \cdot 3-0 \cdot 5$, and well modelled by Domgörgen \& Mathis (1994). It is not clear whether the lower value is due to a less dilute radiation field, or whether features of some models of Sokolowski designed to raise the value, such as a very high upper IMF cutoff, do not apply in these cases.

HeI is detected in NGC 891, but, as in the Reynolds layer, the weakness of $\mathrm{HeI} / \mathrm{H} \alpha$ implies a much softer spectrum than indicated by $[\mathrm{N} \mathrm{II}] / \mathrm{H} \alpha$, and also softer than expected for a population of ionising stars (Rand 1996). A measurement of the gas temperature would help in understanding the forbidden line strengths, but only upper limits exist from the non-detection of the $[\mathrm{N} \mathrm{II}] \lambda 5755$ line: $13,000 \mathrm{~K}$ (east side) and 10,000 K (west side).

Veilleux et al. (1995) have formed an $[\mathrm{N} \mathrm{II}] / \mathrm{H} \alpha$ map of NGC 3079, where again there is a general increase with distance from HII regions, reaching similar values as in NGC 891.

Golla, Dettmar, \& Domgörgen (1996) present long-slit spectra of NGC 4631, and again find an increase of $[\mathrm{N} \mathrm{II}] / \mathrm{H} \alpha$ and $[\mathrm{S} \mathrm{II}] \lambda 6716 / \mathrm{H} \alpha$ with height from the plane. However, the rate of increase of the two ratios with $z$ is about the same (both running from a minimum of about $0 \cdot 13$ in the plane to a maximum of about 0.5 at $z=1 \mathrm{kpc}$ in their slit position ' $\mathrm{C}$ '), whereas their models predict a stronger trend in $[\mathrm{S} \mathrm{II}] / \mathrm{H} \alpha$ than in $[\mathrm{NII}] / \mathrm{H} \alpha$ (because of a more dramatic change in the predominant ionisation state of S between HII regions and diffuse gas). The solution of this discrepancy is not clear, but again it points towards a departure from existing models.

Finally, Ferguson, Wyse, \& Gallagher (1996) present $\mathrm{H} \alpha+[\mathrm{N} \mathrm{II}],[\mathrm{S} \mathrm{II}]$ and $[\mathrm{O} \mathrm{II}] \lambda 3727$ images of NGC 55 , and find that both $[\mathrm{SII}] / \mathrm{H} \alpha+[\mathrm{NII}]$ and $[\mathrm{O} \mathrm{II}] / \mathrm{H} \alpha+[\mathrm{N} \mathrm{II}]$ increase with height from the plane (like $\mathrm{N}$ and $\mathrm{S}$, O becomes increasingly singly ionised with greater distance from the HII regions). The latter ratio shows a very strong contrast, from about $0 \cdot 1$ in the midplane to as high as 2 in the halo. Again, such high values are hard to explain.

Clearly, more work needs to be done to constrain the sources of ionisation and heating. One approach is to model a combination of photoionised gas with shock-ionised gas using various shock speeds, and turbulent mixing layers of various temperatures, as has been done for the much brighter diffuse emission of irregulars by Martin (1996). While such composite models may be able to explain the observations, one still desires a physical scenario which joins these several processes together. For instance, in a model where the diffuse halos are fed by superbubble and chimney activity, what sort of shocks are predicted to run through the halo gas as a result, what sort of mixing interfaces should exist, and how might line ratios change with environment?
As for extra sources of non-ionisation heating, possibilities include photoelectric heating from dust grains (Reynolds \& Cox 1992) and the dissipation of turbulence (Minter \& Balser 1997). The latter authors include a heating rate from turbulence derived from scintillation observations in a photoionisation model of the DIG, and can thereby explain simultaneously $[\mathrm{S} \mathrm{II}] / \mathrm{H} \alpha,[\mathrm{NII}] / \mathrm{H} \alpha$, and $[\mathrm{HeI}] / \mathrm{H} \alpha$ at low $z$ in the Reynolds layer if the composite stellar temperature is low enough.

\section{Acknowledgments}

I would like to thank the organisers for a stimulating and well-run workshop, and the referee, R. Walterbos, for helpful comments.

Bland-Hawthorn, J., \& Jones, D. H. 1998, PASA, 15, 44 Bland-Hawthorn, J., Freeman, K. C., \& Quinn, P. J. 1997, ApJ, 490, 143

Bregman, J. N., \& Houck, J. C. 1997, ApJ, 485, 159

Bregman, J. N., \& Pildis, R. A. 1994, ApJ, 420, 570

Cassinelli, J. P., et al. 1995, ApJ, 438, 932

Dahlem, M., Dettmar, R.-J., \& Hummel, E. 1994, A\&A, 290,384

Dahlem, M., Lisenfeld, U., \& Golla, G. 1995, ApJ, 444, 119

Dettmar, R.-J. 1990, A\&A, 232, L15

Dettmar, R.-J. 1992, Fund. Cosmic Phys., 15, 143

Dettmar, R.-J., \& Schulz, H. 1992, A\&A, 254, L25

Domgörgen, H., Dahlem, M., \& Dettmar, R.-J. 1996, A\&A, 313,96

Domgörgen, H., \& Mathis, J. S. 1994, ApJ, 428, 647

Donahue, M., Aldering, G., \& Stocke, J. T. 1995, ApJ, 450, L45

Dove, J. D., \& Shull, J. M. 1994, ApJ, 430, 222

Duric, N., Irwin, J., \& Bloemen, H. 1998, A\&A, 331, 428

Ferguson, A. M. N., Wyse, R. F. G., \& Gallagher, J. S. 1996, AJ, 112, 2567

Ferrara, A., Bianchi, S., Dettmar, R.-J., \& Giovanardi, C. 1996, ApJ, 467, L69

Golla, G., Dettmar, R.-J., \& Domgörgen, H. 1996, A\&A, 313,439

Golla, G., \& Hummel, E. 1994, A\&A, 284, 777

Heiles, C., Koo, B.-C., Levenson, N. A., \& Reach, W. T. 1996, ApJ, 462, 326

Hummel, E., Sancisi, R., \& Ekers, R. D. 1984, A\&A, 133, 1

Irwin, J. A. 1994, ApJ, 429, 618

Irwin, J. A., \& Seaquist 1990, ApJ, 353, 469

Keppel, J. W., Dettmar, R.-J., Gallagher, J. S., \& Roberts, M. S. 1991, ApJ, 374, 507

Madden, S. C., et al. 1993, in Evolution of Galaxies and Their Environment, Contributed Papers, ed. D. Hollenbach, J. M. Shull \& H. A. Thronson (NASA-CP3190), p. 173

Martin, C. L. 1996, PhD thesis, Univ. of Arizona

Mathis, J. S. 1986, ApJ, 291, 24

Miller, W. W., \& Cox, D. P. 1993, ApJ, 484, 133

Minter, A., \& Balser, D. 1997 ApJ, in press

Norman, C. A., \& Ikeuchi, S. 1989, ApJ, 345, 372

Pildis, R. A., Bregman, J. N., \& Schombert, J. M. 1994, ApJ, 427, 160

Rand, R. J. 1997, in Gas Disks in Galaxies, ed. J. M. van der Hulst (Dordrecht: Kluwer), p. 105

Rand, R. J. 1996, ApJ, 462, 712

Rand, R. J. 1997, ApJ, 474, 129

Rand, R. J., Kulkarni, S. R., \& Hester, J. J. 1990, ApJ, 352, L1 (RKH) 
Rand, R. J., Kulkarni, S. R., \& Hester, J. J. 1992, ApJ, 396,97

Reynolds, R. J., \& Cox, D. P. 1992, ApJ, 400, L33

Reynolds, R. J., \& Tufte, S. L. 1995, ApJ, 439, L17

Reynolds, R. J., Tufte, S. L., Haffner, L. M., Jaehnig, K., \& Percival, J. W. 1998, PASA, 15, 14

Rice, W., et al. 1988, ApJS, 68, 91

Sciama, D. W. 1995, MNRAS, 276, L1

Shapiro, P. R., \& Benjamin, R. A. 1993, in Star Formation, Galaxies, and the Interstellar Medium, ed. J. Franco, F. Ferrini \& G. Tenorio-Tagle (Cambridge Univ. Press), p. 275

Slavin, J. D., Shull, J. M., \& Begelman, M. C. 1993, ApJ, 407, 83
Sokolowski, J. 1994, preprint

Swaters, R. 1994, Undergraduate thesis, Univ. of Groningen Veilleux, S., Cecil, G., \& Bland-Hawthorn, J. 1995, ApJ, 445,152

Vogler, A., Pietsch, W., \& Kahabka, P. 1996, A\&A, 305, 74

Walterbos, R. A. M. 1998, PASA, 15, 99

Walterbos, R. A. M., \& Braun, R. 1996, in ASP Conference Proceedings 106, ed. E. Skillman (San Francisco: ASP), p. 1

Wang, Q. D., Walterbos, R. A. M., Steakley, M. F., Norman, C. A., \& Braun, R. 1995, ApJ, 439, 176

Wyse, R. F. G., Ferguson, A. M. N., Gallagher, J. S., \& Hunter, D. A. 1998, PASA, 15, 118 